\title{
A New OOK Wake-Up Receiver with Sampling Technique
}

\author{
Zhiyuan Tao, Tao Ma, Jicheng Zhang \\ \{zhiyuantao@stu.xidian.edu.cn,tma@xidian.edu.cn,jichzhang@stu.xidian.edu.cn\} \\ Xidian University, Xian, 710071
}

\begin{abstract}
In this paper, we proposed OOK wake-up receiver (WuR) with the sampling technique. In this method, the power consumption of RF envelope detector, which is the most power hungry component in the receiver, is greatly reduced by the signal-level duty cycling (or sampling), and the original OOK modulated IF signal is recovered by a designed active IF bandpass filter, which consume only micro power. Using this novel method, a new wake-up receiver with the high sensitivity and the low power consumption is designed and implemented. According to our test, the lowest input RF power that the OOK modulated signal can be recovered is $-70 \mathrm{dBm}$, which is almost same as the sensitivity of envelope detector LT5538. Meanwhile, the power consumption of our WuR is about $310 \mu \mathrm{W}$.
\end{abstract}

Keywords: Wake-up receiver, sampling, RF envelope detector.

\section{Introduction}

Wireless sensor networks (WSNs) have been recognized as an enabling technology for a large variety of applications, including smart homes and cities, agriculture, transportation, health and fitness, entertainment, and structural health monitoring [1]. Reducing the communication power consumption of WSN nodes is important, as the radio transceiver is one of the components with the highest power consumption. Optimizing the power consumption of the wireless transceiver can free up power budget to add much more functionality. In addition, since the battery size is the decisive factor for the entire size of battery, operated systems, lowpower circuits could enable smaller batteries and lead to miniaturization required by many applications such as wearable WSNs, medical body area networks and implantable devices.

To establish communication, two radios (the receiver and the transmitter) need to be synchronized as a message can be received only if the radio is in its listening state, and idle listening consumes significant power. Hence, a significant design effort is required to alleviate this power waste. To reduce communication power consumption, several techniques have been proposed [2]-[7] for lowering or eliminating the power wasted due to idle listening of the transceiver. Duty cycling is a common technique to reduce the idle mode energy consumption which consists of switching from listening mode to sleep mode [2]. However, while duty cycling helps save power, it can severely limit the network reactivity as the radios are OFF (or in the sleep state) and they cannot receive messages. Different from general duty cycling receiver, bit-level duty cycling receiver samples only a portion of each transmitted bit to reduce the power consumption of RF front end [8]. The paper [9-10] designed a logarithmic power detector based WuR with the bit sampling technique, obtaining sensitivity of $-77 \mathrm{dBm}$. In this 
paper, we proposed a signal-level sampling wake-up receiver, whose power consumption can be dynamically adjusted by MCU. Furthermore, we proposed an active IF filter to recovery the original continuous IF signal from the samples of the output of RF envelope detector. The attenuation caused by sampling can be alleviated by an IF amplifier, which consumes only micro watt power due to its low frequency. Therefore, the overall power of WuR can also keep in several micro watts by the duty cycling (or sampling) the high-power component RF envelope detector. According to our test, the lowest input RF power that the proposed WuR can successfully recovery the OOK modulated signal is about $-70 \mathrm{dBm}$, which is almost same as the sensitivity of envelope detector LT5538. Meanwhile, we could calculate the power consumption of WuR is about $310 \mu \mathrm{W}$.

The rest of paper is organized as follows: Section 2 gives the description of the proposed WuR with sampling, mathematically analyze attenuation of the output of the IF bandpass filter. Section 3 presented a simulation design of IF filter, and conducted the hardware experimental test to evaluate the performance of the proposed WuR. Finally, the conclusion remarks were given in Section 4.

\section{The proposed OOK WuR}

\subsection{The description of the proposed OOK WuR}

We proposed a new WuR with sampling technique, which is shown in Fig.1.

The OOK modulated RF signal is chosen as the input of our WuR. In our WuR, the envelope detector is enabled only short duration $\tau$ every period T. Then the output of our envelope detector is the samples of original demodulated OOK signal, which is shown in Fig. $2 \mathrm{~b}$. After the bandpass filter with a proper setting and the comparator, the original OOK signal can be recovered.

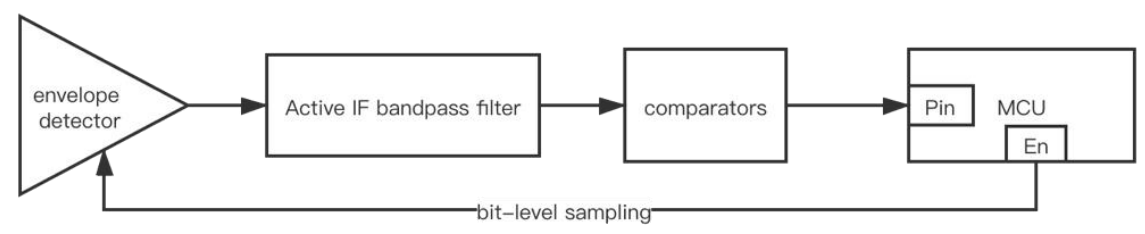

Fig. 1. The architecture of WuR.

By signal-level sampling the envelope detector, which is the most power hungry component, the overall power of WuR is greatly reduced. After an bandpass filter and an IF amplifier, the original OOK signal is recovered from the samples with limited power consumption. Therefore, compared with the tuned RF receiver, the proposed wake-up receiver is able to demodulate OOK signal with much lower power consumption.

\subsection{The Theoretical Analysis}

Let $\mathrm{S}(\mathrm{t})$ denote the periodic square wave signal with period $\mathrm{T}, \mathrm{S}_{\mathrm{p}}(\mathrm{t})$ denote the periodic pulse signal with period $T_{p}$ and pulse width $\tau$. The output of envelope detector can be expressed as follows. 


$$
\begin{gathered}
S_{S}(t)=S(t) \times S_{p}(t) \\
S_{p}(t)=\left\{\begin{array}{rr}
1 & n T-\frac{\tau}{2}<t<n T+\frac{\tau}{2} \\
0 & n T+\frac{\tau}{2}<t<(n+1) T-\frac{\tau}{2}
\end{array}\right.
\end{gathered}
$$

Let $F_{s}(w)$ be the Fourier transform of $S_{s}(t), S(w)$ denote the Fourier transform of $\mathrm{S}(\mathrm{t})$.

$$
F_{S}(w)=\frac{1}{2 \pi} S(w) \otimes F\left[S_{p}(t)\right]
$$

Where $F\left(S_{p}(t)\right)$ are

$$
F\left[S_{p}(t)\right]=2 \pi \sum_{n=o}^{N} \frac{\tau}{T} S a\left(\frac{\pi n \tau}{T_{p}}\right) \delta\left(w-n \frac{2 \pi}{T_{p}}\right)
$$

Combining (3) and (4), we have:

$$
F_{S}(w)=\frac{\tau}{T}(S(w))+\sum_{n=1}^{N} S a\left(\frac{\pi n \tau}{T_{p}}\right) S\left(w-n \frac{2 \pi}{T_{p}}\right)
$$

When $1 / T_{p} \geq 2 / T$, by using a perfect bandpass filter, we can recover $F_{f}(w)$.

$$
F_{f}(w) \approx \frac{\tau}{T} S(w)
$$

Thus, by using a proper bandpass filter, we could recover original signal. As we can see from equation (6), the strength of the recovered signal is related with duty cycle of the signal $\mathrm{S}_{\mathrm{p}}(\mathrm{t})$, which is the ratio between $\tau$ and $\mathrm{T}$. By decreasing this ratio, the average power of envelope detector is reduced, however at the same the amplitude of the recovered signal is also decreased. Nevertheless we could use low power IF amplifier to enlarge the recovery signal since its frequency is not high. 


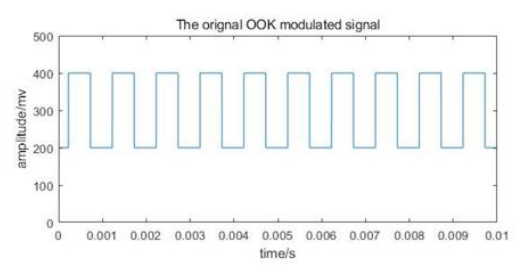

a. The output of the envelope detector without sampling

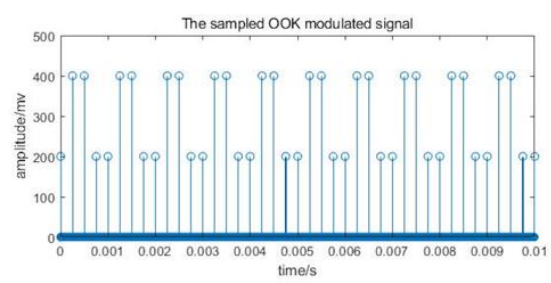

b. The output of the envelope detector with sampling

Fig. 2. The output signal of the envelope detector comparison between with and without sampling.

\section{The Simulation and Experimental Results}

We use TINA-TI software simulation tools provided by TI company [13] to simulate the active filter. We design a bandpass filter with cut-off frequency of $3 \mathrm{Khz}$ to filter out the alias frequency of the sampled signal. The designed active bandpass filter is shown in fig. 3 .

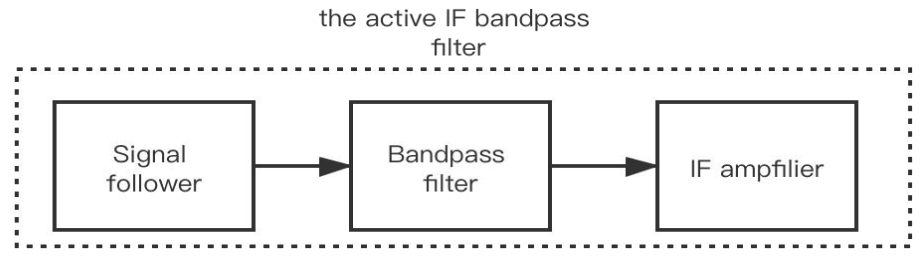

Fig. 3. The active IF bandpass filter.

It is worth noting that the output impedance of envelope detector in our WuR changes over time. The output impedance of LT5538 is $150 \Omega$ when it is enable and is $30 \mathrm{~K} \Omega$ when it is off [11]. These changes can significantly impact the characteristic of designed filter. In order to eliminate this influence we add a signal follower in front of IF active filter to stabilize the input impedance of designed bandpass filter. Its frequency characteristic of our designed filter is shown in Fig. 4 . We can see that it has $-3 \mathrm{db}$ gain at point $\mathrm{A}$ where the frequency is $7 \mathrm{HZ}$ and point $\mathrm{B}$ where the frequency is $3 \mathrm{KHZ}$. The purpose of filtering out low frequency signals below $7 \mathrm{HZ}$ is to avoid excessive DC voltage caused from the envelope detector. On the other hand, filtering out low frequency signals avoids DC voltage changes due to communication distance changes. The purpose of filtering out frequency signals above $3 \mathrm{KHz}$ is to recover IF signal.

Furthermore, we conducted the hardware experiment to evaluate the proposed idea. We adopted LT5538 as the RF envelope detector, which has the sensitivity of $-71.5 \mathrm{dBm}$ at 880 MHZ. It consumes $29 \mathrm{~mA}$ current at active stage, while consumes $1 \mu \mathrm{A}$ at disabled stage. LMV551 is chosen to compose bandpass filters. It consumes $49 \mu \mathrm{A}$ at $3 \mathrm{~V}$. MSP430FR5969 is chosen as microcontroller in our WuR. It consumes $210 \mu \mathrm{A}$ at $3 \mathrm{~V}$ when it is active, and only $0.4 \mu \mathrm{A}$ at low power mode 3 where only auxiliary clock is on. One of its IO pin is connected to the enable port of LT5538 to control its working stage. The output of filter is connected to another pin in our microcontroller. The measurement setup shows in Fig.5. 


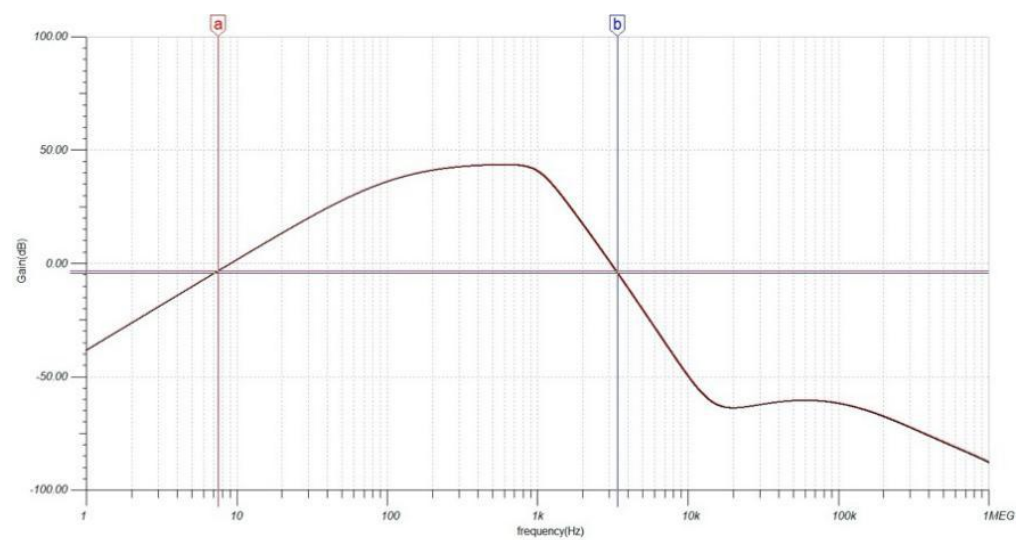

Fig. 4. The frequency characteristic of active filter

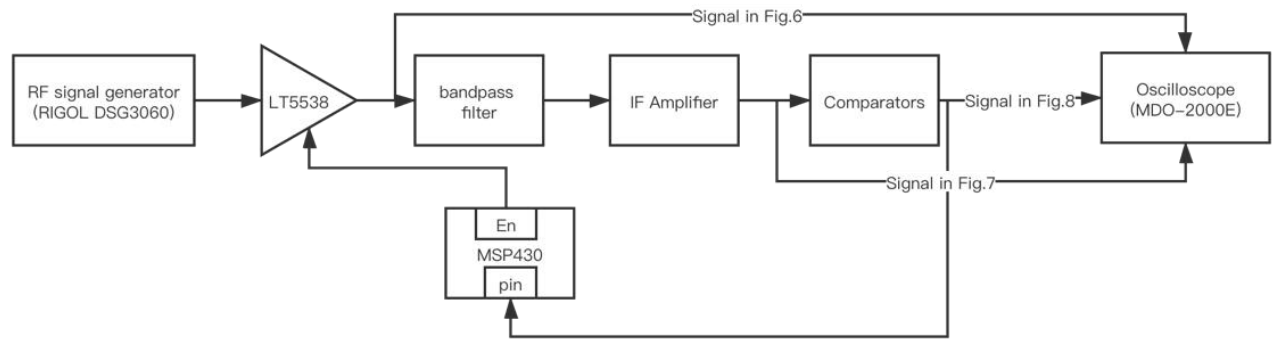

Fig. 5. The measurement setup.

In this simulation, the RF signal generator creates the $1 \mathrm{kbps}$ OOK modulated signal. We samples the envelope detector LT5538 by a rectangular pulse signal with a frequency of $4 \mathrm{kHz}$ and a pulse width of $1 \mu \mathrm{s}$, which is shown in Fig.6. It means that power consumption of RF envelope detector is $1 / 100$ of the original power. This sampled signal is selected as the input of our designed filter. Fig. 7 shows output of the filter. Finally, the recovered signal is shown in Fig.8. The designed filter successfully recovered the original signal from its samples as analyzed earlier in this article.

As you can see from our test, OOK signal is successfully recovered. Now we analyze the power of the proposed WuR in our implementation. In this paper we only consider the power of RF front end, since it is most power hungry part of the receiver. Let $\mathrm{P}_{\text {ea }}$ and $\mathrm{P}_{\mathrm{ei}}$ be the power of envelope detector in active stage and inactive stage, $P_{f}$ be the power of filter, $P_{a}$ be the power of comparator. We assume that $n=\frac{T}{T_{p}}$. Then the power of our WuR can be expressed as:

$$
P_{a l l}=\frac{n \tau}{T} \times P_{e a}+\left(1-\frac{n \tau}{T}\right) P_{e i}+P_{f}+P_{a}
$$

If we set $\tau=1 \mu \mathrm{s}, \mathrm{n}=4$ and we select the hardwares in Table 1 , we could easily calculate the power consumption of $\mathrm{WuR}$ is about $310 \mu \mathrm{W}$. 
According to our test, the lowest input RF power that the proposed WuR can successfully recovery the OOK modulated signal is about $-70 \mathrm{dBm}$, which is almost same as the sensitivity of envelope detector LT5538.

Table 1. The hardware powers.

\begin{tabular}{cccc}
\hline & Hardware & Power(active) & Power(idle) \\
\hline Active filter & LMV551[12] & $111 \mu \mathrm{W}$ & $111 \mu \mathrm{W}$ \\
Envelope detector & LT5538[11] & $90 \mathrm{~mW}$ & $3 \mu \mathrm{W}$ \\
Comparator & TLV3691[14] & $152 \mathrm{nW}$ & $152 \mathrm{nW}$ \\
\hline
\end{tabular}

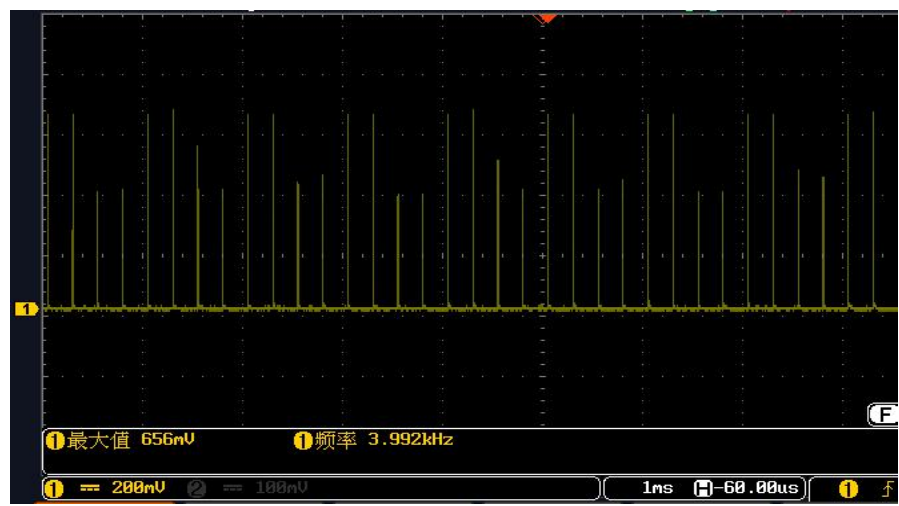

Fig. 6. The sampled OOK signals. (-60dbm input)

\section{Conclusion}

In this paper we proposed wake-up receiver with the sampling technique. In this method, the RF frontend component power consumption is greatly reduced by signal-level sampling. On the other hand, the original IF OOK modulated signal is recovered by a designed active bandpass filter with limited power consumption. By this method, the overall power consumption of the receiver is greatly reduced without lowering the sensitivity. Using this new method, we implemented the OOK wake-up receiver with the off-the-shelf components. According to our experimental results, the implemented wake-up receiver can consume only less than $310 \mathrm{uW}$ and achieve a $-70 \mathrm{dBm}$ sensitivity. 


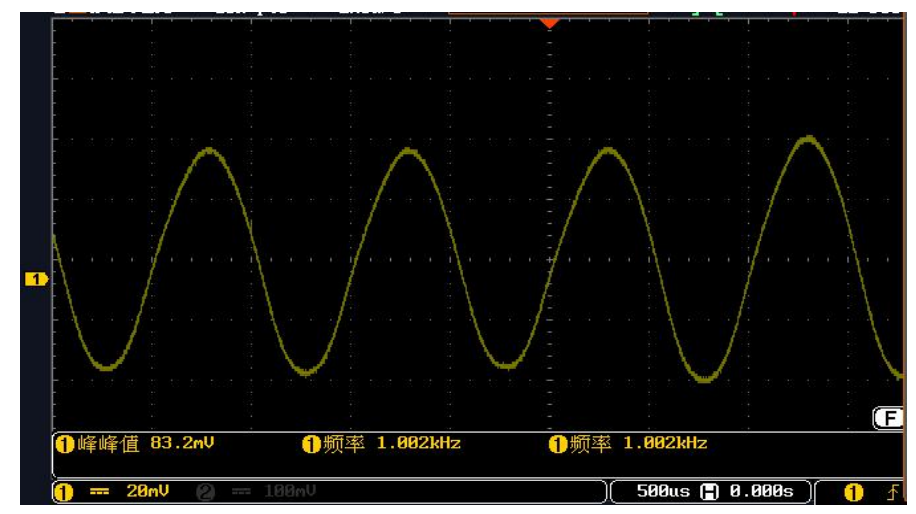

Fig. 7. The output of the filter amplification. (-60dbm input)

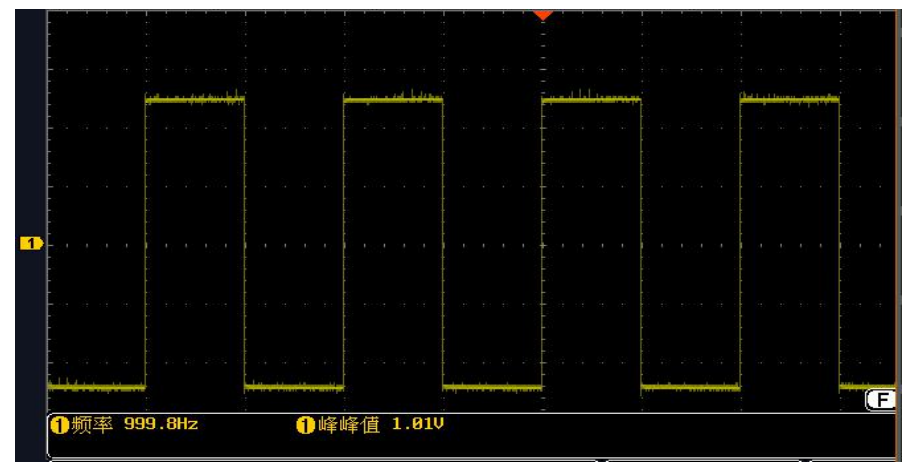

Fig. 8. The recovered signal. (-60dbm input)

\section{References}

[1] A. Willig.: Recent and emerging topic in wireless industrial communications: A selection. vol. 4, no. 2, pp. 102-124, IEEE Trans. Ind. Informat (2008)

[2] I. Demirkol, C. Ersoy, and E. Onur.: Wake-up receivers for wireless sensor networks: Benefits and challenges, vol. 16, no. 4, pp. 88-96, IEEE Wireless Commun, US (2009).

[3] V. Jelicic, M. Magno, D. Brunelli, V. Bilas, and L. Benini.: Analytic comparison of wake-up receivers for WSNs and benefits over the wakeon radio scheme, pp. 99-106, in Proc. 7th ACM Workshop Perform. Monit. Meas. Heterogen, Wireless Wired Netw (2012)

[4] M. Magno, D. Boyle, D. Brunelli, E. Popovici, and L. Benini.: Ensuring survivability of resourceintensive sensor networks through ultra-low power overlays, vol. 10, no. 2, pp. 946-956, IEEE Trans. Ind. Informat, US (2014)

[5] H. Ba et al.: Passive RFID-based wake-up radios for wireless sensor networks, pp. 113-129, in Wirelessly Powered Sensor Networks and Computational RFID. New York, NY, USA,Springer(2013)

[6] A. Castagnetti, A. Pegatoquet, T. N. Le, and M. Auguin.: A joint dutycycle and transmission power management for energy harvesting WSN, vol. 10, no. 2, pp. 928-936, IEEE Trans. Ind. Informat, US (2014). 
[7]D. Spenza, M. Magno, S. Basagni, L. Benini, M. Paoli, and C. Petrioli.: Beyond duty cycling: Wakeup radio with selective awakenings for longlived wireless sensing systems, pp. 522-530. IEEE Conf. Comput. Commun. (INFOCOM), Proc (2015).

[8] Xiao Y. Wang, Rajeev K. Dokania, and Alyssa B. Apsel.: A Crystal-Less Self-Synchronized BitLevel Duty-Cycled IR-UWB Transceiver System. VOL. 60, NO. 9, IEEE transactions on circults and systems (2013)

[9] T. Ma.: A Bit Sampled Wake-Up Receiver with Logarithmic Detector Architecture. pp. 445-449. International Conference on Cyber-Enabled Distributed Computing and Knowledge Discovery, Nanjing (2017)

[10] T. Ma.: Single-Carrier-Detection-Based Wake-Up Receiver Via Compressive Sensing. vol. 22, no. 5, pp. 954-957, IEEE Communications Letters (2018)

[11] LT5538 datasheet, LinearTechnology.http://cds.linear.com/docs/en/datashee/5538f.pdf. retrieved in 2018.

[12] LMV551 datasheet, Texas Instruments. http://www.ti.com/lit/ds/symlink/lmv551.pdf. retrieved in 2016.

[13] TINA-TI. SPICE-based analog simulation program. http://www.ti.com/tool/TINATI?keyMatch=DOWNLOAD\%20TINA\&tisearch=Search-EN-everything

[14] TLV3691 datasheet, Texas Instruments. http://www.ti.com/lit/ds/symlink/tlv3691.pdf retrieved in 2014. 